\title{
Spectral interferometry and reflectometry used for characterization of a multilayer mirror
}

\author{
Petr Hlubina, ${ }^{*}$ Jiří Luňáček, Dalibor Ciprian \\ Department of Physics, Technical University Ostrava, \\ 17. listopadu 15, 70833 Ostrava-Poruba, Czech Republic \\ *Corresponding author: petr.hlubina@vsb.cz
}

\begin{abstract}
Compiled April 16, 2009
A white-light spectral interferometric technique is used to retrieve a relative spectral phase and group delay of a multilayer mirror from the spectral interferograms recorded in a dispersive Michelson interferometer. The phase retrieval is based on the use of a windowed Fourier transform in the wavelength domain and characterization of the multilayer mirror is completed by a three-step measurement of the reflectance spectrum of the mirror in the same interferometer. (C) 2009 Optical Society of America

OCIS codes: $120.3180,120.2650,120.5700,230.4040$.
\end{abstract}

Characterization of a multilayer mirror involves determination of the complex reflection coefficient that includes the mirror reflectance and phase change on reflection from the mirror. Moreover, the group delay (GD) introduced when a pulse is reflected by the mirror is also of great importance. There exist many different methods reported in the literature to measure these quantities. Interferometric methods, including the timedomain and spectral-domain implementations, belong to the most powerful techniques of measuring the phase changes and the GDs characterizing optical components $[1,2,3,4,5,6]$. In this Letter a white-light spectral interferometric method $[7,8,9]$ for characterization of multilayer mirrors is presented. The technique, which is rather simple, utilizes a Michelson interferometer with one of its mirrors replaced by a multilayer mirror. From the spectral interferograms recorded by a fiber-optic spectrometer a relative spectral phase and a GD (the first derivative of the phase) characterizing the mirror are retrieved. The phase retrieval is based on the use of a windowed Fourier transform [8]. Moreover, in the Michelson interferometer the reflectance spectrum of the mirror is also measured by a three-step procedure [9].

Let us consider the mutual interference of two beams from a broadband source at the output of a slightly dispersive Michelson interferometer with a cube beamsplitter of the effective thickness $t_{\text {eff }}[7,8,9]$ when mirror 1 of the interferometer is replaced by a multilayer mirror as shown in Fig. 1. We assume that the mirror is characterized by a complex reflection coefficient

$$
r(\lambda)=\sqrt{R(\lambda)} \exp \left[\mathrm{i} \delta_{r}(\lambda)\right]
$$

where $R(\lambda)$ is the wavelength-dependent reflectance and $\delta_{r}(\lambda)$ is the wavelength-dependent phase change on reflection. Moreover, we assume that the geometrical path lengths of the light rays in dispersive glass of the beamsplitter are not the same for both interferometer arms so that the beamsplitter can be represented by an ideal beamsplitter and a plate of the same dispersion and of the thickness $t_{\text {eff }}$ (see Fig. 1). The complex reflection coefficient $r_{2}(\lambda)$ of mirror 2 is represented by a relation similar to that of Eq. (1) with $R_{2}(\lambda)$ and $\delta_{2}(\lambda)$ as the reflectance and the phase change on reflection, respectively. The wavelength-dependent optical path difference (OPD) $\Delta(\lambda)$ between two beams in the Michelson interferometer is given by

$$
\Delta(\lambda)=2 L+2 n(\lambda) t_{\mathrm{eff}}-\lambda\left[\delta_{r}(\lambda)-\delta_{2}(\lambda)\right] /(2 \pi),
$$

where $2 L$ is the difference of path lengths between the interfering beams in the air whose dispersion is neglected and $n(\lambda)$ is the wavelength-dependent refractive index of the beamsplitter material.

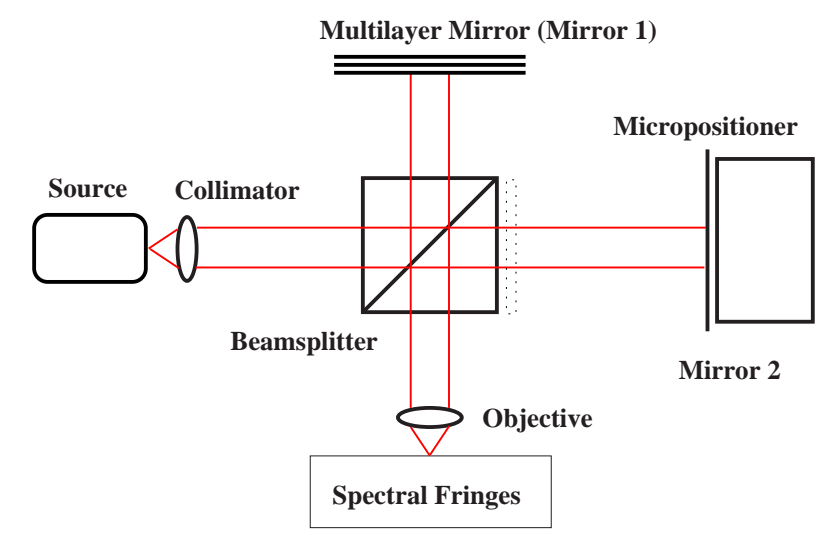

Fig. 1. (Color online) Experimental setup with a Michelson interferometer to measure a multilayer mirror.

The procedure for the interferometric measurement [7, 9] of a relative spectral phase is based on recording of the spectral interferograms in the dispersive Michelson interferometer and retrieving the absolute OPD $\Delta(\lambda)$. The absolute OPD $\Delta(\lambda)$ is used to construct for a chosen mirror position $L=L_{0}$ the relative spectral phase $\delta(\lambda)$ given by the relation

$$
\delta(\lambda)=(2 \pi / \lambda)\left[2 L_{0}+2 n(\lambda) t_{\mathrm{eff}}-\Delta(\lambda)\right]+\delta_{2}(\lambda) .
$$


To compensate for the phase change $\delta_{2}(\lambda)$, a next measurement step with the reference sample used instead of the multilayer mirror needs to be applied [9].

The procedure for the reflectance measurement is with the arm of mirror 2 blocked and it consists of three steps: first, with the source blocked, the background spectrum $I_{b k g}(\lambda)$ is measured; second, with a reference sample used instead of mirror 1 , the reference reflection spectrum $I_{\text {ref }}(\lambda)$ is measured; third, with the multilayer mirror used instead of the reference sample, the reflection spectrum $I_{\text {meas }}(\lambda)$ of the mirror is measured. The absolute reflectance $R(\lambda)$ of the multilayer mirror is given by

$$
R(\lambda)=\frac{I_{m e a s}(\lambda)-I_{b k g}(\lambda)}{I_{r e f}(\lambda)-I_{b k g}(\lambda)} R_{r e f}(\lambda),
$$

where $R_{r e f}(\lambda)$ is the theoretical reflectance of the reference sample which can be computed by use of experimental data available from optical handbooks [10].

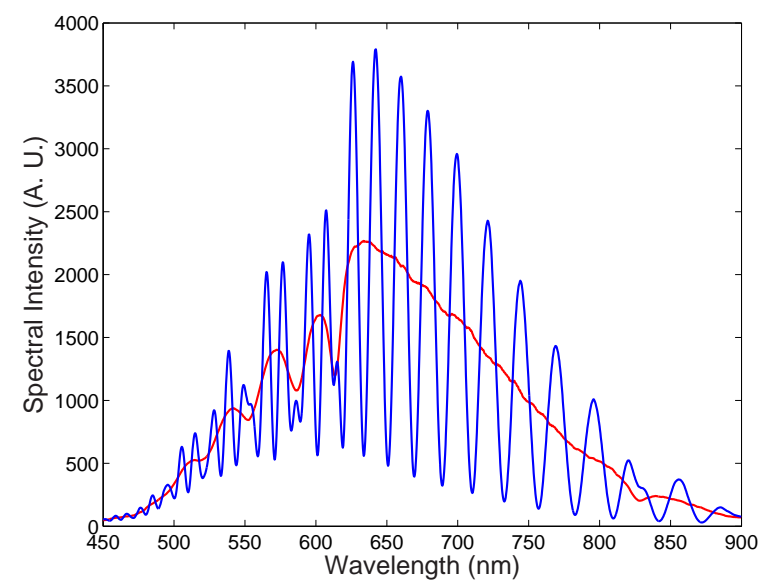

Fig. 2. (Color online) Background spectrum and channeled spectrum recorded for a multilayer mirror in the setup shown in Fig. 1.

The experimental setup used for measurement of a multilayer mirror is shown schematically in Fig. 1 and it consists of a white-light source: a halogen lamp HL-2000 (Ocean Optics) with launching optics, an optical fiber and a collimating lens, a bulk-optic Michelson interferometer with a cube beamsplitter made of BK7 optical glass, a sample of multilayer dielectric mirror, metallic mirror 2 connected to a micropositioner, a microscope objective, micropositioners, a read optical fiber, a fiberoptic spectrometer S2000 (Ocean Optics), an A/D converter and a personal computer. The spectrometer has a spectral operation range from 350 to $1000 \mathrm{~nm}$.

The procedure used to record spectral intensities, from which a relative spectral phase $\delta(\lambda)$ of a multilayer mirror can be retrieved, comprises two steps. In the first step, the spectral intensity $I(\lambda)$ with spectral interference fringes (channeled spectrum) is recorded by the spectrometer. To obtain the channeled spectrum, one manually translates the micropositioner with mirror 2 until spectral fringes appear. The position of the micropositioner corresponds to the adjusted OPD $\Delta(\lambda)$. In the second step, the background spectral intensity $I_{0}(\lambda)$, i. e., the intensity without spectral interference fringes, is recorded by the spectrometer. To obtain the background spectrum, one manually translates the micropositioner until spectral fringes disappear completely. From the two spectral intensities, the spectral signal $S(\lambda)=I(\lambda) / I_{0}(\lambda)-1$ is evaluated which is used to retrieve the relative spectral phase $\delta(\lambda)$ by using a suitable processing procedure [7]. To reach the finite spectral signal, the background spectrum needs to be above the zero. Figure 2 shows examples of the spectral intensities with and without the spectral fringes recorded for the sample of a multilayer dielectric mirror in the wavelength range from 450 to $900 \mathrm{~nm}$. There is apparent effect of the multilayer mirror causing the abrupt changes in spectral intensities not present for standard metallic mirrors [8].

We use a simple and effective procedure for retrieving the relative spectral phase $\delta(\lambda)$ from the spectral signal $S(\lambda)$. We process the spectral signal $S(\lambda)$ by a windowed Fourier transform [8] to retrieve the overall spectral phase $\Phi(\lambda)$ with the ambiguity of $m 2 \pi$, where $m$ is an integer. This processing of the spectral intensities gives the overall spectral phase with a higher accuracy than a standard Fourier-transform processing. Moreover, its implementation is easier in comparison with a wavelet-based processing that determines the overall spectral phase from the ridge of the wavelet transform of the recorded channeled spectrum [11]. Next, we use a procedure [7] of the absolute retrieval of the adjusted OPD based on the linear dependence of the OPD $\Delta(\lambda)$ on the refractive index $n(\lambda)$ of the beamsplitter material which is know (BK7 glass). The slope of the linear dependence is given, as indicates Eq. (2), by the effective thickness $t_{\text {eff }}$ of the beamsplitter, which can be determined by a similar procedure from the spectral signal measured in the same Michelson interferometer but with two identical metallic mirrors [8]. The linear dependence gives also an estimate of the mirror position $L=L_{0}$, which is needed in the evaluation of the relative spectral phase $\delta(\lambda)$ according to Eq. (3). The phase change $\delta_{2}(\lambda)$ due to mirror 2 is measured in the same setup with the reference sample (a bare Si wafer) [9]. Other estimate of the mirror position $L=L_{0}$ results from the approximation $\delta\left(\lambda_{0}\right) \approx 0$, which means that the relative spectral phase of a multilayer mirror is approximately zero at a center wavelength $\lambda_{0}$ of the bandpass of the mirror reflectance spectrum. Fortunately, there is no problem with determining the center wavelength $\lambda_{0}$ because the reflectance spectrum $R(\lambda)$ can be measured by a three-step procedure presented above in the same setup as the relative spectral phase $\delta(\lambda)$. Figure 3 shows the reflectance spectrum $R(\lambda)$ recorded for the sample of a multilayer mirror. Once again, there is apparent effect of the multilayer mirror causing the abrupt changes in the reflectance spectrum not present for standard metallic mirrors [9]. The reflectance is nearly 1 for the mirror 
spectral bandpass (from 619 to $820 \mathrm{~nm}$ ) and the corresponding central wavelength is $719.5 \mathrm{~nm}$. The relative spectral phase $\delta(\lambda)$ of the mirror satisfying the condition $\delta\left(\lambda_{0}\right)=0$ is also shown in Fig. 3. It is characterized by both the abrupt phase changes at the wavelengths with minimum reflectances and the steep phase decrease within the mirror spectral bandpass. It should be noted that the retrieved relative spectral phase $\delta(\lambda)$ is much greater than the phase change $\delta_{2}(\lambda)$ due to mirror 2 [9]. Moreover, the spectral phase retrieved for the first estimate of the mirror position $L_{0}$ is shifted by a constant in comparison with that retrieved for the second estimate of the mirror position $L_{0}$.

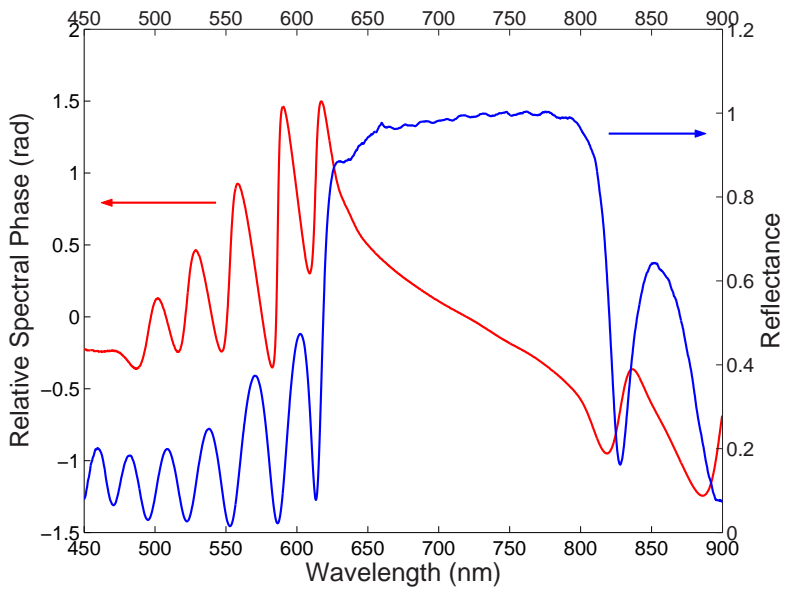

Fig. 3. (Color online) Retrieved relative spectral phase and measured reflectance spectrum of a multilayer mirror.

Finally, Fig. 4 shows the GD of the multilayer mirror evaluated as the derivative of the relative spectral phase $\delta(\omega)$ with respect to the angular frequency $\omega=2 \pi c / \lambda$, where $c$ is the speed of light in vacuum. This figure clearly shows spikes in the GD that correspond to minima in the reflectance spectrum of the multilayer mirror.

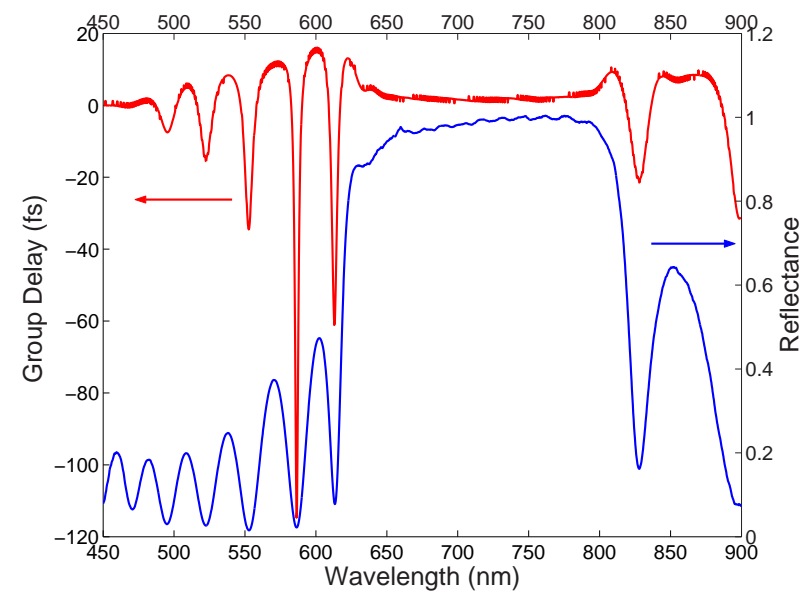

Fig. 4. (Color online) Retrieved group delay and measured reflectance spectrum of a multilayer mirror.
In conclusion, a simple white-light spectral interferometric technique for measuring the relative spectral phase and the reflectance spectrum of a multilayer mirror at the same time has been presented. The technique utilizes a cost-effective instrumentation with a Michelson interferometer and a fiber-optic spectrometer. The spectrometer records the background and channeled spectra to obtain the spectral signal whose processing by a windowed Fourier transforms gives a relative spectral phase. The feasibility of the technique has been demonstrated in measuring the GD and the reflectance of a sample of a multilayer dielectric mirror. We confirmed that the wavelength positions of extrema of both quantities agree well. The use of the technique can be extended, for example, for measuring the GD of narrow-bandpass filters employing high-resolution fiber-optic spectrometers or optical spectrum analyzers.

The research has been partially supported by the Ministry of Education, Youth and Sports of the Czech Republic through grant MSM6198910016.

\section{References}

1. W. H. Knox, N. M. Pearson, K. D. Li, and C. A. Hirlimann, Opt. Lett. 13, 574 (1988).

2. M. Beck and I. A. Walmsley, Opt. Lett. 15, 492 (1990).

3. S. Diddams and J.C. Diels, J. Opt. Soc. Am. B 13, 1120 (1995).

4. A. P. Kovacs, K. Osvay, Z. Bor, and R. Szipocs, Opt. Lett. 20, 788 (1995).

5. T. D. Noe, Appl. Opt. 41, 3183 (2002).

6. K. Ogawa, Appl. Opt. 45, 6718 (2006).

7. P. Hlubina, D. Ciprian, J. Luňáček, and M. Lesňák, Opt. Express 14, 7678 (2006).

8. P. Hlubina, J. Luňáček, D. Ciprian, R. Chlebus, Opt. Commun. 281, 2349 (2008).

9. P. Hlubina, J. Luňáček, D. Ciprian, and R. Chlebus, Appl. Phys. B 92, 203 (2008).

10. E. Palik, Handbook of Optical Constants of Solids (Academic, San Diego, Calif., 1985), Vol. I.

11. D. Reolon, M. Jacquot, I. Verrier, G. Brun, and C. Veillas, Opt. Express 14, 12744 (2006). 